\title{
Incorporation of Load Induced Thermal Strain in Finite Element Models
}

\author{
A. Law, M. Gillie, P. Pankaj
}

Load induced thermal strains (LITS) are an integral part of the behaviour of concrete in fire; their existence has been well documented and modelled by different researchers. More thorough representation of LITS is needed to accurately represent their plastic constituents in finite element models. This paper develops a technique to allow the evolution of LITS in accordance with the rules developed in several academic models. The technique is implemented with a simple Drucker-Prager yield surface and the results assessed.

Keywords: Load induced thermal strain, finite element, concrete, structures in fire, yield surface.

\section{Introduction}

Load induced thermal strain (LITS) is an integral part of the behaviour of concrete in fire. The existence of LITS has been well documented and modelled by different researchers. It is vital that this strain development is correctly represented in structural models, as the locked in strains due to LITS constituents are significant. Current methods of modelling LITS involve incorporating the strains into constitutive curves. This approach allows the total strains developed due to LITS to be simply included in a finite element analysis. More thorough representation is needed to accurately represent the plastic components in loading directions, and the total strains in non-loading directions. This paper presents a technique to allow the evolution of LITS in accordance with the rules developed in several academic material models [1-3]. The technique is implemented with a simple Drucker-Prager yield surface and the results assessed.

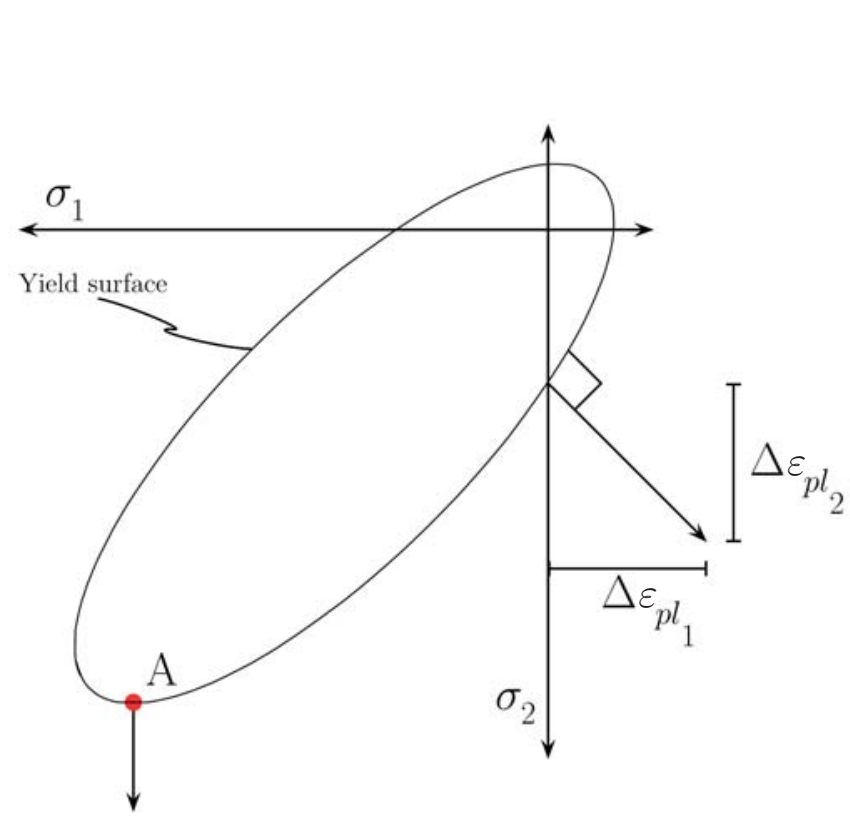

\section{Current methods}

Inclusion of LITS in a concrete constitutive curve is a convenient way of representing LITS in finite element analyses. It allows the modeller to make the LITS constituents temperature dependent and stress dependent - through the use of multiple curves and by giving strains for different stresses respectively. A number of models are available from different sources and for different concretes [1-4]. Failure to represent LITS will result in the modeller not modelling the strains developed in the material accurately, thereby giving an excessively stiff structure. In fact, it could be argued that since LITS is an integral part of concrete behaviour, a modeller failing to include it will not be modelling concrete but some other, non-physical, material.

Once the total strains caused by LITS have been represented, one can then think about the division between elastic and plastic strains. It has been observed that the largest LITS

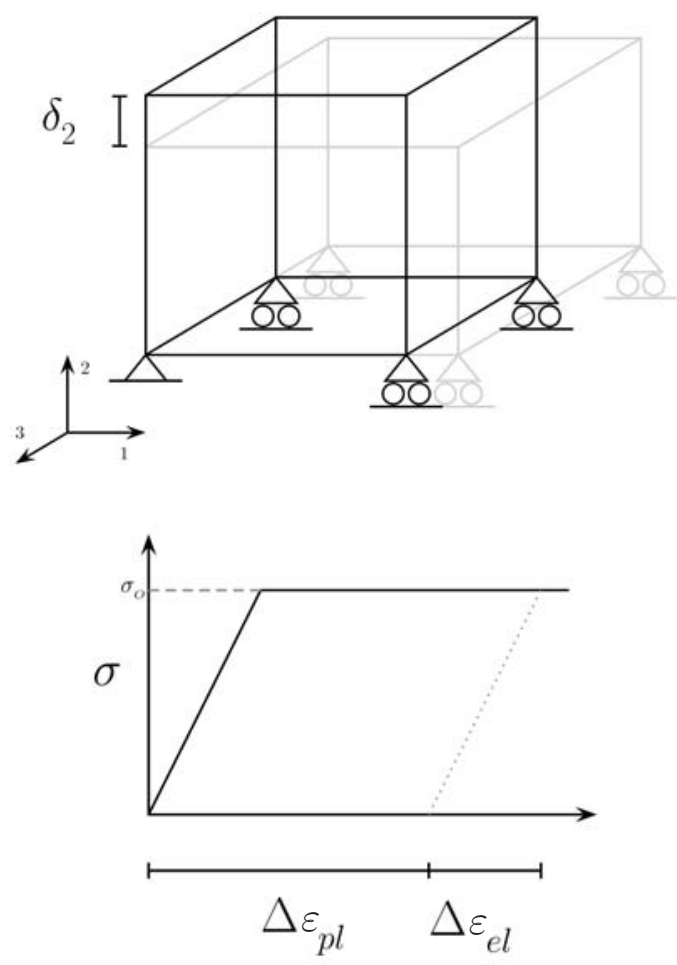

Fig. 1: Plastic flow, and model setup 
constituents are irrecoverable [5], i.e. they are plastic strains. Therefore, to accurately model these plastic strains it is necessary to determine the elastic modulus of the material as a function of temperature. If the modulus is too stiff, the plastic strains will be overestimated; too soft, and they will be underestimated. The correct modelling of plastic strain constituents becomes increasingly important as a structure cools, since the plastic strains will induce greater tension on strain reversal.

Some authors have presented their material models in parts, allowing the user to build the strain constituents into the full curve. The elastic modulus is, therefore, a precisely identifiable constituent of the material model and can be included in a structural model as such; henceforth, this will be termed the "actual" modulus. Other material data such as that presented in the Eurocode do not specify the value of the elastic modulus. In this case, extra care must be taken to represent the strain components accurately. Where the elastic modulus is the initial gradient of the constitutive curve, this will be termed the "apparent" modulus.

\section{Multiple dimensions}

The primary focus for research has been on total and plastic strains in the direction of loading. However, attention must also be paid to the non-loading directions. Depending on the model in use, failure to carefully consider the elastic modulus of the material will result in unrepresentative plastic strains, unexpected strains in the non-loading directions, or a mixture of both. The potential for these effects to manifest themselves can be demonstrated by a simple example.

\subsection{Simple example}

Consider a small cube of concrete, subject to a displacement controlled loading in principle direction 2, but free to move in the transverse directions with a Drucker-Prager yield surface and a perfectly plastic material behaviour, as shown in Fig. 1. The associative isotropic flow rule (used here for simplicity) dictates that once the yield surface is reached, plastic strain must occur in a direction orthogonal to the yield
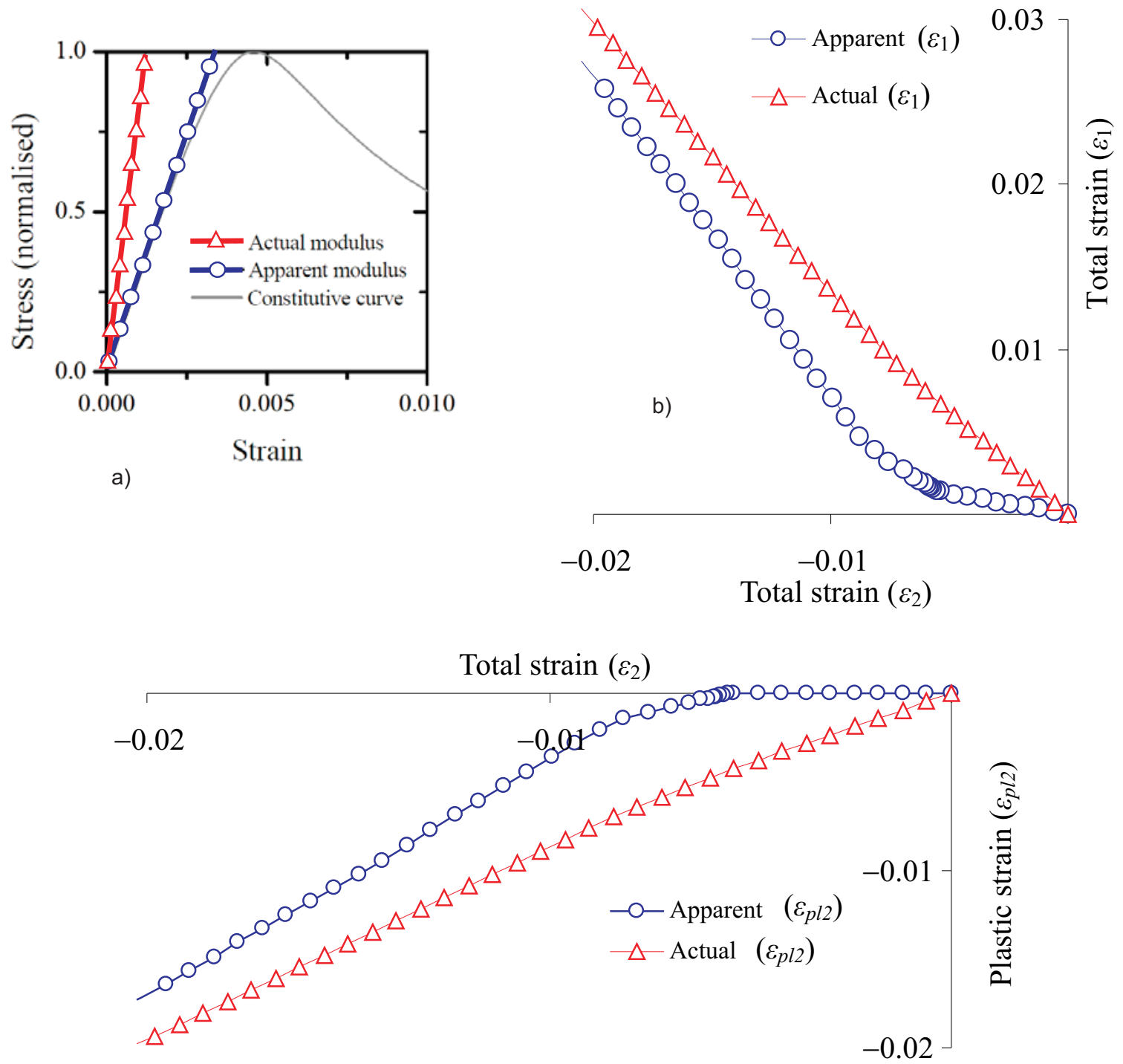

c)

Fig. 2. The same constitutive curve with different elastic moduli gives different lateral deformations and direct plastic strains 
surface in stress space. This means that plastic strains are induced in directions other than the one in which the load is applied.

Since the location of trial stress is a function of the elastic modulus, the implications of this for the implementation of LITS via a constitutive curve are significant. The inclusion of LITS whether implicitly (with an "apparent" elastic modulus) or explicitly (with an "actual" elastic modulus) will result in a proportion of that LITS becoming active in the transverse directions. The magnitude of the extra strain would depend on the stress state of the material, and on the degree of plasticity developed in the principle direction. For example: should the element described above be at a stress state at point A, no plastic strains would be induced in the 1-direction.

In the case of the apparent modulus, a large proportion of the extra transverse strain may be elastic; while in the case of the actual modulus, the major constituent of the incremental strain would be plastic.

The impact of this difference is demonstrated below, using the Drucker-Prager yield criterion with a constitutive curve corresponding to that of the $200{ }^{\circ} \mathrm{C}$ Terro [2] LITS curve. This temperature was used as there is a significant difference

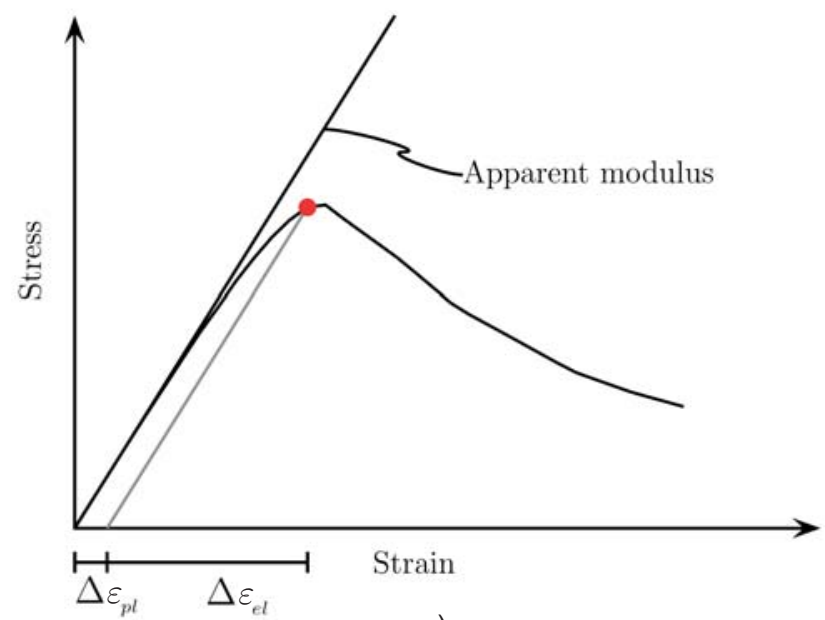

a)

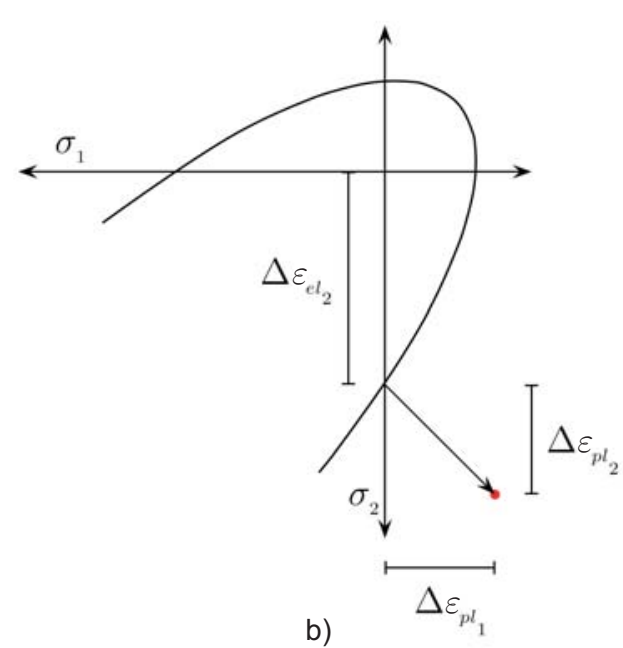

Fig. 3: Calculation of plastic and elastic strains: a) hardening with apparent modulus, b) corresponding strains between the actual and apparent moduli, but the temperature is not too extreme. Two different models were created, each with a different elastic modulus - apparent or actual - but with the same constitutive curve (Fig. 2a). The numerical models consisted of a single cubic finite element, restrained at the base in the 2-direction (but free to displace in the 1 and 3-directions) and were strained in the 2-direction. The corresponding deformations and plastic strains were recorded.

Fig. $2 b$ shows the total strains in the lateral deformation direction. The strains in the 2-direction (i.e. the direction of strain control) are the same for both of the models. In the unrestricted directions, however, there are significant differences in the total strains, particularly in the inelastic phase of the constitutive model. The origin of these differences can be clearly seen from Fig. 2c. In the "apparent" model the plastic strains do not develop until much later in the deformation process. The "actual" model on the other hand - because of the difference between the elastic modulus and the shape of the constitutive curve - activates the plastic strain constituents immediately. This difference in plastic strain is entirely due to the activation of the flow rule at a much lower stress. Conse-

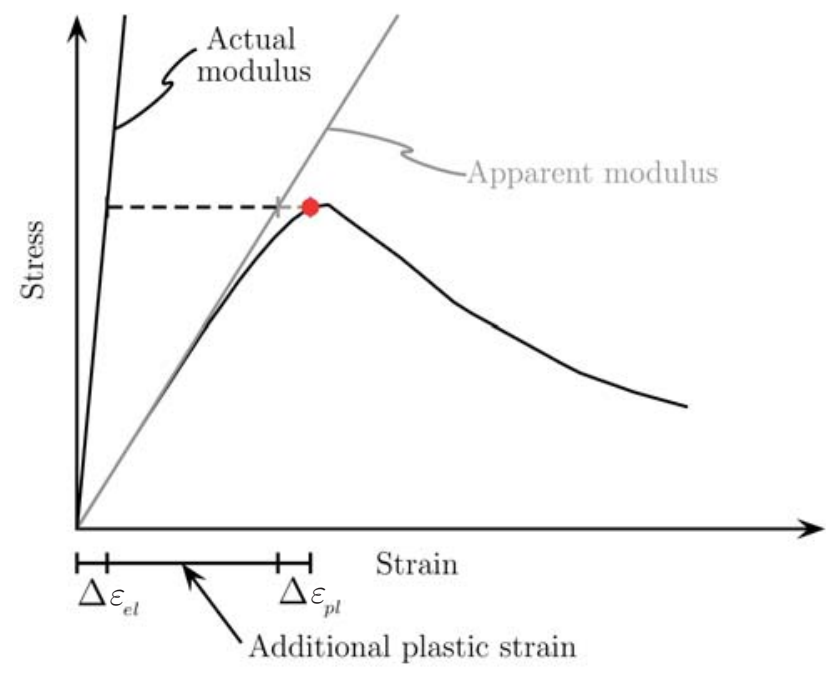

a)

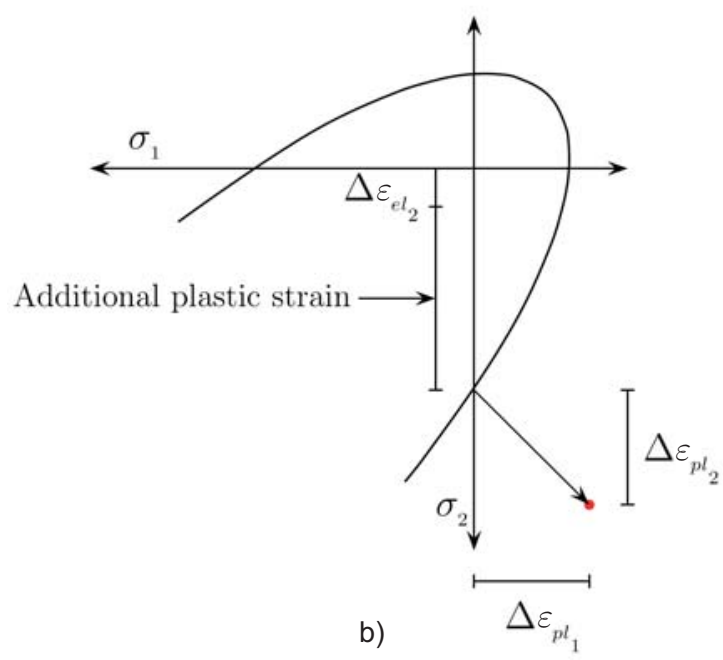

Fig. 4: Redistribution of strains due to the difference between actual modulus and apparent modulus: a) redistribution found using actual modulus, b) corresponding strains 
quently, though the plastic strain in the loading direction is what would be expected from using the "actual" modulus in the constitutive curve, the impact of this approach can be clearly seen in the non-loading directions.

Since equations to represent LITS are all functions of temperature and direct stress, the use of either the apparent or the actual modulus is inadequate if one wants to model the plastic strains accurately, whilst limiting the lateral deformations.

\section{The embedded modulus}

To allow the modelling of LITS to be more representative, a new method is proposed for the inclusion of LITS in the constitutive model while avoiding the transverse strain issue outlined above. The Drucker-Prager yield criterion and plasticity equations are solved in a two step method: first, the elastic strains and corresponding plastic strains are calculated using the apparent modulus and the normal solution methods (Fig. 3$)$; secondly, the elastic $\left(\varepsilon_{\text {ell }}\right)$ and plastic $\left(\varepsilon_{\text {pl1 }}\right)$ strains are recalculated using the actual modulus (Fig. 4). As such, the actual modulus is embedded within the solution procedure. This second stage can expressed simply as:

$$
\varepsilon_{\mathrm{el} 1}=\frac{\sigma}{E_{\mathrm{em}}}
$$

where $E_{e m}$ is the embedded actual modulus and $\sigma$ is the stress calculated from the previous solution. Since:

$$
\varepsilon_{\mathrm{el} 0}+\varepsilon_{\mathrm{pl} 0}=\varepsilon_{\text {total }},
$$

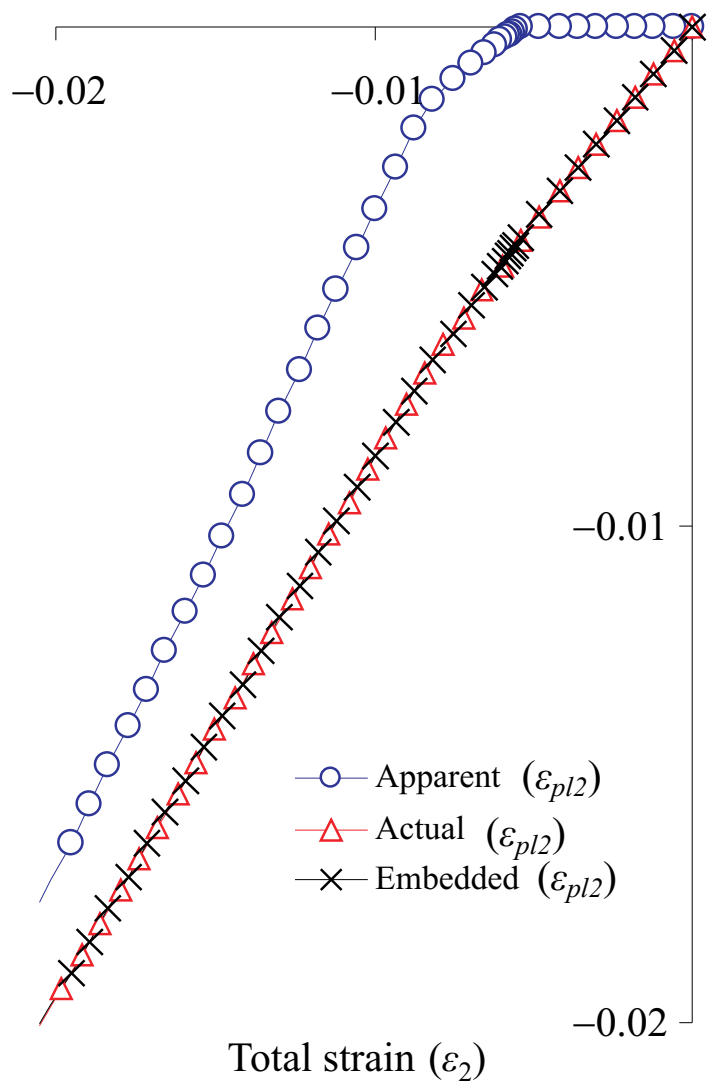

where $\varepsilon_{\mathrm{el} 0}$ and $\mathrm{a} \varepsilon_{\mathrm{pl} 0}$ are the original elastic and plastic strains, and $\varepsilon_{\text {total }}$ is the total strain. The new plastic strain can be directly calculated from:

$$
\varepsilon_{\mathrm{pl} 1}=\varepsilon_{\text {total }}-\varepsilon_{\text {el } 1} .
$$

The new plastic and elastic strains are then used in the subsequent analysis. The equivalent plastic strain is not, however, changed. Consequently, the strains developed in the transverse directions are in line with those that would occur when using an apparent modulus, but the plastic strains developed in the principle direction are as would be expected from using the actual modulus. It should also be noted that where plastic strain has occurred, but the yield function is found to be negative (i.e. the total strain is reduced), the corresponding elastic stresses must be recalculated using the embedded modulus. Otherwise, the redistributed strains would be reabsorbed into the elastic region on return to zero stress.

A Drucker-Prager model was created [6-12] which incorporated this method of modification by the embedded modulus. A model with an apparent elastic modulus and an embedded actual modulus was subjected to the previously described test. The results were compared with the previous models (Fig. 5).

The total lateral strains experienced by the "embedded" material are the same as those experienced by the "apparent" material. Equally, the total plastic strain experienced in the loading direction is the same as those experienced by the "actual" material. Thus, a fully plastic, transient strain constituent has been included in the model without affecting the

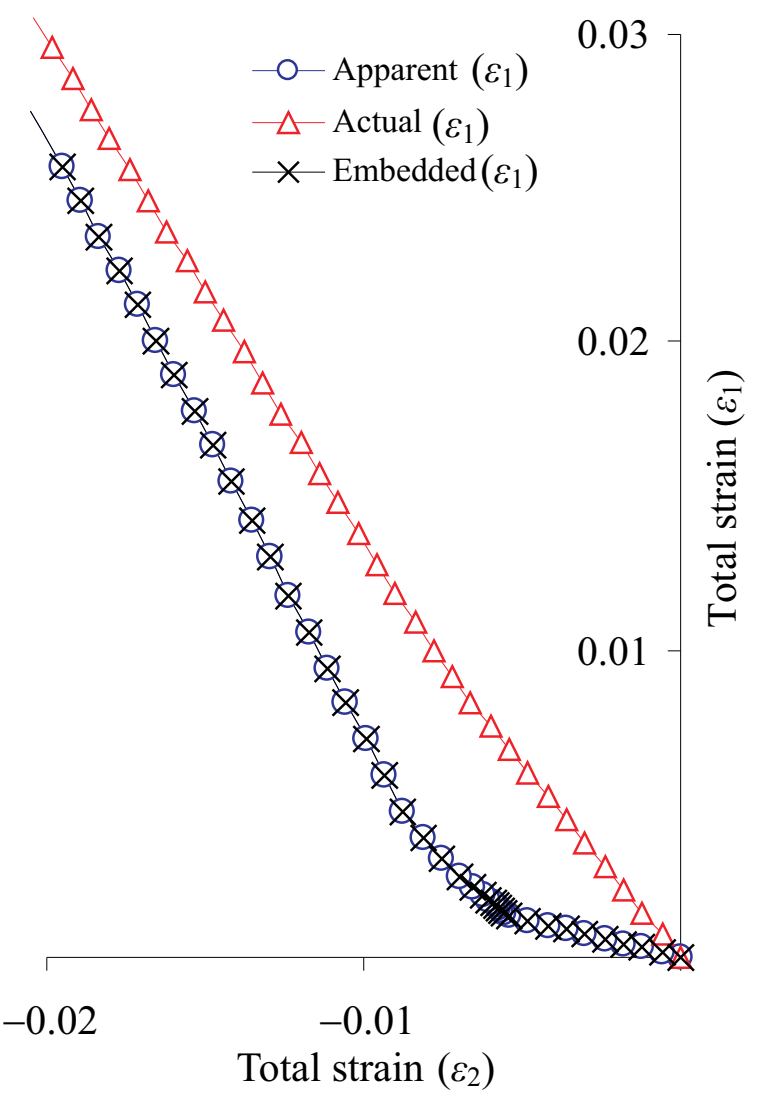

Fig.5. Comparison of two stage approach with results from original models 
deformations in the non-loading directions. This allows the plastic LITS effect to be successfully modelled uni-axially and in proportion to the applied stress in the way stated in the governing LITS equations.

\section{Conclusion}

There are several conclusions to be drawn from this study:

- There are significant differences between a constitutive curve which includes LITS, and a full constitutive model which accurately represents LITS components.

- Inclusion of the plastic strains by means of an "apparent" modulus is useful in one dimension; however, plastic flow rules cause unwanted strains to develop laterally when more than one dimension is considered.

- Use of a two step model with an "apparent" modulus, and an embedded "actual" modulus within the material model is one approach which can be used to correctly model the plastic strain due to the LITS equations, while allowing the strain in the lateral directions to be modelled correctly. This model has been demonstrated in the case of an element deformed uniaxially.

The authors of this work would like to gratefully thank the project sponsors; BRE Trust, and the EPSRC.

\section{References}

[1] Anderberg, Y., Thelandersson, S.: Stress and Deformation Characteristics of Concrete, 2 - Experimental Investigation and Material Behaviour Model. Sweden, University of Lund, 1976.

[2] Terro, M. J.: Numerical Modeling of the Behaviour of Concrete Structures in Fire. ACI Structural Journal, Vol. 95 (1998), p. 183-193.

[3] Nielsen, C. V., Pearce, C. J., Bicanic, N.: Theoretical Model of High Temperature Effects on Uniaxial Con- crete Member under Elastic Restraint. Magazine of Concrete Research, Vol. 54 (2002), p. 239-249.

[4] EN1992-1-2: Design of Concrete Structures - Part1-2: General Rules - Structural Fire Design, 1992.

[5] Khoury, G. A., Grainger, B. N., Sullivan, P. J. E.: Transient Thermal Strain of Concrete: Literature Review, Conditions within Specimen and Behaviour of Individual Constituents. Magazine of Concrete Research. Vol. 37 (1985), p. 131-144.

[6] Calladine, C. R.: Plasticity for Engineers: Theory and Applications. Chichester: Horwood Publishing, 2000.

[7] Crisfield MA: Non-Linear Finite Element Analysis of Solids and Structures. Chichester: Wiley, 1991.

[8] Crisfield MA: Non-Linear Finite Element Analysis of Solids and Structures: Advanced Topics. Chichester: Wiley, 1997.

[9] Cook, R. D., Malkus, D. S., Plesha, M. E., et al.: Concepts and Applications of Finite Element Analysis. Chichester: Wiley, 2002.

[10] Hill, R.: The Mathematical Theory of Plasticity. Oxford: Oxford University Press, 1950.

[11] Pankaj, P.: Finite Element Analysis in Strain Softening and Localisation Problems, in Department of Civil Engineering. Swansea: University College of Swansea, 1990.

[12] Zienkiewicz, O. C.: The Finite Element Method (ed. 3). London: McGraw-Hill, 1977.

\footnotetext{
Angus Law

Martin Gillie

Pankaj Pankaj

The University of Edinburgh

BRE Centre for Fire Safety Engineering

Edinburgh, UK
} 